\title{
Electroneuromyographic indices of respiratory muscles in patients with chronic obstructive pulmonary disease
}

\begin{abstract}
The functional state of respiratory muscles and diaphragm was assessed in 28 patients with chronic obstructive pulmonary disease of 2, 2-3, 3 degrees of severity according to electroneuromyography at the age of 49 to 65 years. The revealed violations of the functional state of the respiratory muscles and the function of external respiration significantly aggravate the progressive nature of the pathological process in the lungs in COPD
\end{abstract}

Keywords: chronic obstructive pulmonary disease, respiratory diseases. sutures, diaphragm, respiratory function, lungs.

\author{
Volume 4 Issue 6 - 2019
}

\author{
Sadykova GA,' Aripov BS, ${ }^{2}$ Rakhmatullayev \\ $\mathrm{HU},{ }^{3}$ Karimova GV, ${ }^{4}$ Akhmedov $\mathrm{MA}^{3}$ \\ 'Head of a grant by a doctor of medical sciences, prof of the \\ Republican specialized scientific center of Therapy and medical \\ rehabilitation, Uzbekistan \\ ${ }^{2} \mathrm{Head}$ of the department of pulmonology, Candidate of medical \\ sciences, Associate professor of the Republican Specialized \\ scientific center of Therapy and medical, Uzbekistan \\ ${ }^{3}$ Senior researcher the Republican specialized scientific center \\ of Therapy and medical rehabilitation, Uzbekistan \\ ${ }^{4}$ Junior Researcher of the Republican specialized scientific \\ center of Therapy and medical rehabilitation, Uzbekistan
}

Correspondence: Sadykova GA, Head of a grant by a doctor of medical sciences, Prof of the Republican specialized scientific center of Therapy and medical rehabilitation, Uzbekistan, Email glora@yandex.ru

Received: October 09, 2019 | Published: November 06, 2019

\section{Introduction}

In the structure of chronic lung diseases, the most common are chronic obstructive pulmonary diseases (COPD). ${ }^{1}$ One of the rare causes of dyspnea, which cannot be explained by other diseases, is diaphragm dysfunction. ${ }^{2,3}$ An important role in the development of respiratory failure in patients with COPD is played by increasing weakness of the respiratory muscles, mainly the diaphragm. ${ }^{3}$ In addition to the diaphragm, the auxiliary respiratory muscles also participate in the process of ventilation: the scalene, parasternal internal and external intercostal, sternocleidomastoid, trapezoid. ${ }^{4}$

Respiratory musculature dysfunction is a condition in which a decrease in the strength and speed of its contractions occurs as a result of a significant increase in the volume of work performed, ${ }^{4}$ inadequate energy supply, and also the initial non-physiological state of the muscles (NHLBI Work shop Summary, 1990).

Skeletal and respiratory muscles dysfunction is one of the most common systemic effects in patients with chronic obstructive pulmonary disease (COPD). ${ }^{5}$ currently; only pulmonary rehabilitation methods, such as physical training, can change the functional status of patients, improve physical activity by improving the work of skeletal and respiratory muscles.

\section{Purpose of the study}

To detect electroneuromyographic changes in respiratory muscles in patients with chronic obstructive pulmonary disease.

\section{Material and methods}

A study was conducted of the functional state of the respiratory muscles and the pulmonary function test (PFT) in 28 COPD patients aged 49 to 65 years. Among the examined patients, men (77\%) predominated compared with women $(23 \%)$. The first group includes patients with COPD with a 2 and 2-3 severity level - 13 people, patients of the $2^{\text {nd }}$ group - COPD of the 3 rd degree of severity -15 people.

To assess the functional state of the respiratory muscles and diaphragm, electroneuromyography (ENMG) was performed on a Myograph Synapsis Neurotech Russian apparatus. The following modes of operation of the apparatus were used: muscle response (M-response), latency (L-lat). The following were determined: the amplitude $(\mathrm{mV})$ of the M-response of the phrenic and long pectoral nerves: laterally (ms) of the nerves of Phrenicus D et S (region of the sternoclavicular - mastoid muscle); and N. Toracisus longus D et $\mathrm{S}$ (Erb's point). We analyzed a graphic image of the pulse extension rate (SPI-distal).

The study of the function of external respiration was carried out using a desktop computer spirograph Shiller, and a peak flow meter. The following FVD indicators were calculated: FVC (1); FEV 1.0 L; FEV 1.0 FVC -\%; FEF25-75\%; PEF 1/s; MEF 75\%; MEF 50\%; MEF $25 \%$. The peak expiratory flow rate (PSV) was measured according to the generally accepted method. ${ }^{5}$ 


\section{Results}

Patients complained of causeless shortness of breath, decreased exercise tolerance, periodic respiratory failure in sleep, drowsiness, decreased quality of life, and development of respiratory failure. In patients with COPD 2 and 2-3 tbsp. severity revealed moderate clinical symptoms of the disease and mild changes in clinical and laboratory parameters. In this group of patients with COPD, a decrease in the m-response along the right phrenic nerve, signs of moderate dysfunction of the anterior scalene muscle were revealed.
Patients COPD-3CT. the severity had moderate changes in clinical and laboratory data and the results of instrumental examination. An ENMG study of respiratory muscles in patients of the second group revealed a moderate decrease in the amplitude of the muscular response along the left phrenic nerve and long thoracic nerve. The signs of muscular - diaphragmatic changes in the muscles of the chest were revealed (Figure 1).

A moderate increase in latency in the left diaphragmatic and right long chest nerve was revealed in the examined patients (Figure 2).

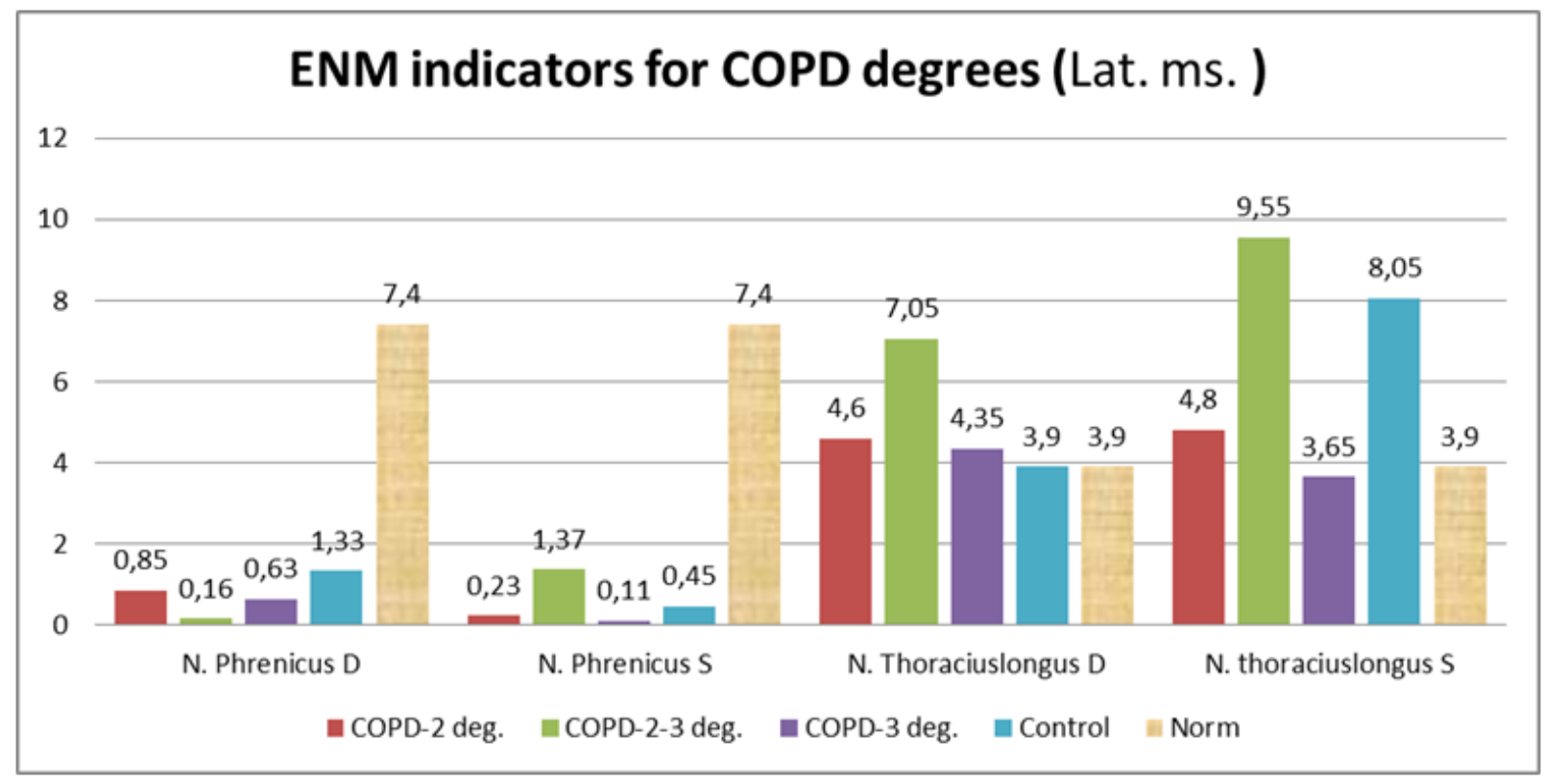

Figure I shows the results of ENMG lateralized (Lat., Ms) in degrees of severity in a comparative aspect with the control group.

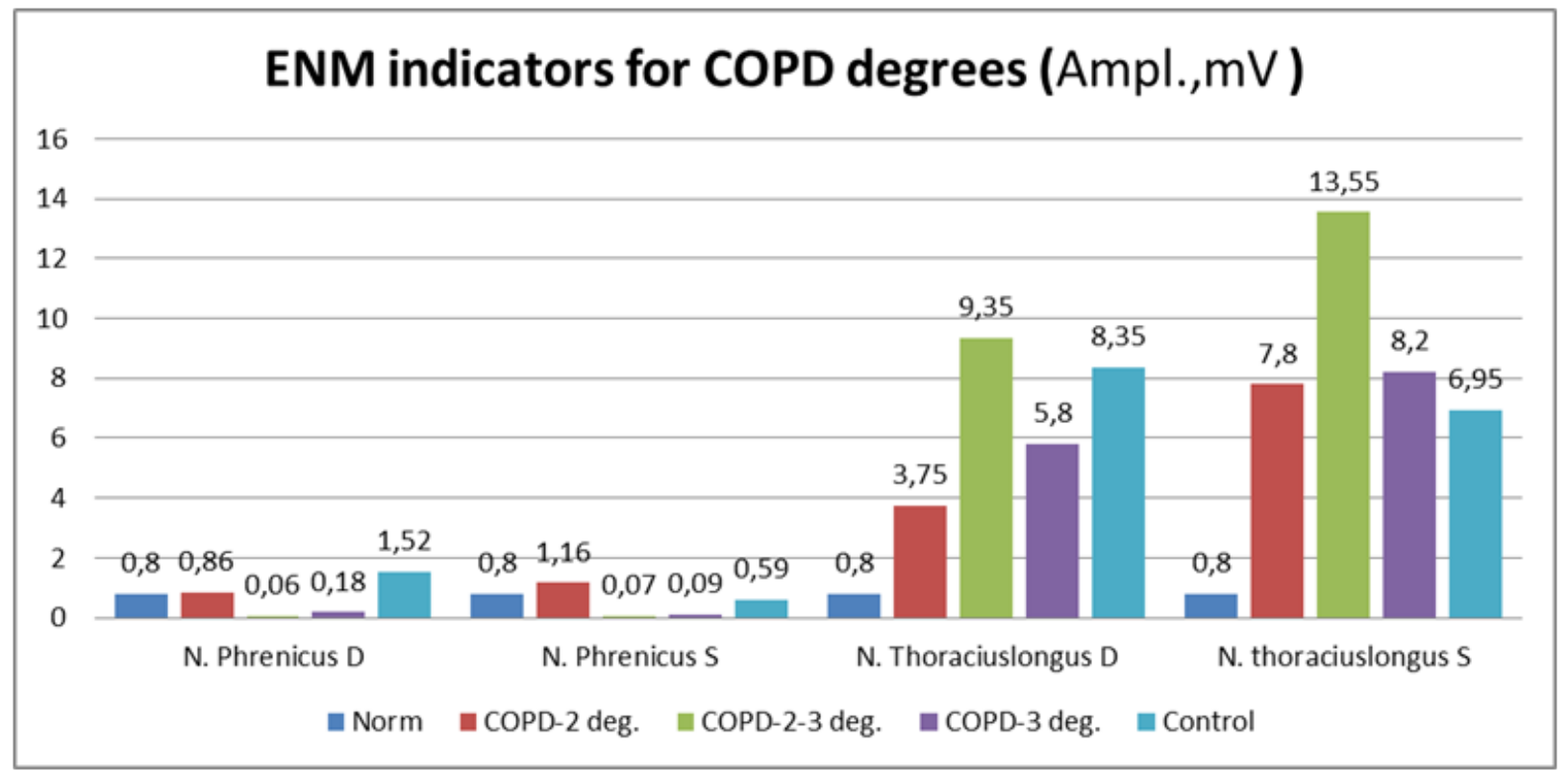

Figure 2 shows the results of ENMG studies in the studied groups according to severity in comparison with the control according to the amplitude of the muscle response (Amp. MV). 
Patients with COPD revealed the same type of changes: the amplitudes of the m-response along the long thoracic nerves from 2 sides were reduced; moderate signs of muscular-dystrophic changes in the anterior dentate muscles of the chest from 2 sides were reduced.

Thus, a moderate decrease in the amplitude of the muscular response along the left phrenic nerve and long thoracic nerve was revealed in patients with COPD. Signs of muscular-dystrophic changes in the muscles of the chest and signs of moderate dysfunction of the anterior scalene muscle. The data obtained confirm the opinion of a number of authors that the thickening of the diaphragmatic muscle is associated with its hyper activation due to increased respiratory load against the background of bronchial obstruction and at the beginning of the disease is functional in nature. ${ }^{6,7}$

In the study of PFT, the majority of the examined patients showed moderate restrictive disorders with a decrease in bronchial patency of the bronchi, as evidenced by PFT 1.0, which were moderately reduced in all patients. Moreover, in 7 patients the decrease was $8-10 \%$ of the proper values, and in 4 patients - more than $13 \%$. FVC (1) was significantly reduced in all observation groups $(\mathrm{p}<0.05)$. A moderate decrease was noted - MEF $50 \%$ to $43.7 \pm 4.3 \%$ (Figure 3 ).

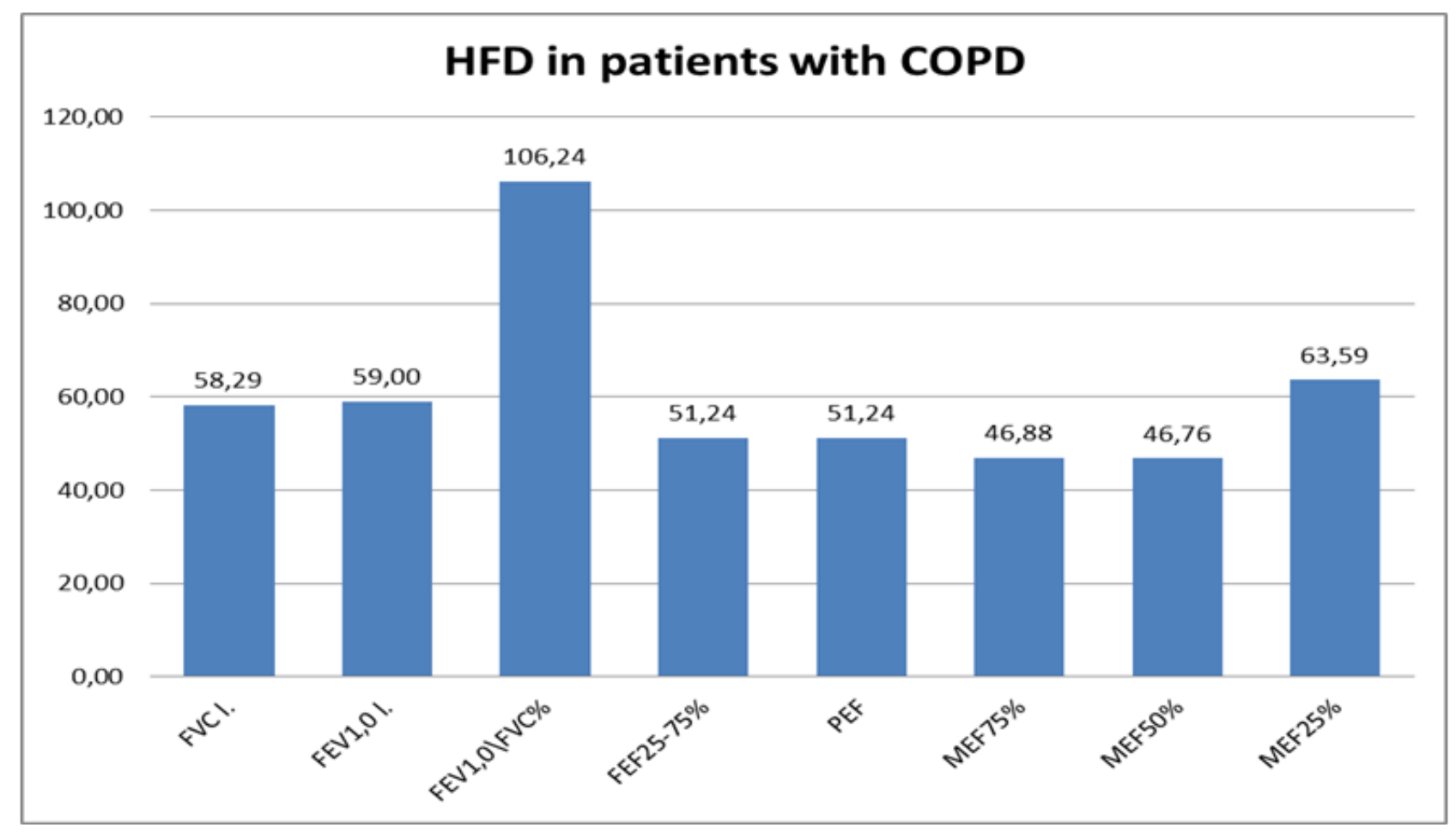

Figure 3 HFD in patients with COPD.

In most patients with COPD 3rd stage pronounced bronchial obstruction was detected, as evidenced by PSV, which was reduced by $20-25 \%(\mathrm{p}<0.05)$ in 21 patients $(60 \%)$, and in 14 patients $(40 \%)$ the peak expiratory flow was reduced by $27-35 \%(\mathrm{p}<0.05)$. FEV1 was reduced (by $25 \%$ at $p<0.05$ ), which indicated the progression of the pathological process in these patients.

The revealed changes indicated an increase in the degree of bronchial obstruction in patients with COPD, this exacerbated the adverse conditions for the functioning of the diaphragm in this category of patients and created the prerequisites for the development and progression of respiratory failure.

Other groups of respiratory muscles are forced to take on a significant share of ventilation work with ineffective contractions of the diaphragm, at the first stages compensating for the "tired" diaphragm, but then the irreversible state of the diaphragm - its "weakness" - occurs. Consequently, the role of other groups of respiratory muscles in the formation of the respiratory act as a whole is much less, and they cannot long-term compensate for the work of a "tired" diaphragm. ${ }^{6,7}$ The obtained results confirm that at the beginning of the pathological process, patients with COPD have a hyper function of the diaphragmatic muscle with an increase in the thickness of the muscle of the diaphragm, a change in the relaxation rate of the muscle of the diaphragm, and a decrease in excursion of the diaphragm during forced breathing. Further progression of the pathological process in the lungs leads to the development and aggravation of $\mathrm{DN}$, which may be due, inter alia, to an increase in dysfunction of smooth muscle structures of blood vessels and bronchi. ${ }^{4}$ Fatigue develops with subsequent weakness of the respiratory muscles due to depletion of the reserves of compensation, atrophic changes begin to prevail, while the tendon part of the diaphragm loses its elasticity. ${ }^{8-10}$

\section{Findings}

1. In patients with COPD, a moderate decrease in the amplitude of the muscular response along the left phrenic nerve and long thoracic nerve was revealed. Signs of muscular-dystrophic changes in the muscles of the chest and signs of moderate dysfunction of the anterior scalene, dentate muscles. 
2. Identified violations of the functional state of the respiratory muscles and the function of external respiration significantly exacerbate the progressive nature of the pathological process in the lungs with COPD.

\section{Acknowledgement}

None.

\section{Conflicts of interest}

The authors declare that there is no conflict of interest.

\section{References}

1. Chuchalin AG. Global strategy for the diagnosis, treatment and prevention of chronic obstructive pulmonary disease (2010 revision)/In: AG Chuchalina, editor. Pulmonology Wedge Recom. 2010:250.

2. Aisanov ZR. Fatigue of the respiratory muscles-issues of diagnosis and treatment. 1987:24.

3. Babaev MA, Birg TM, Bykov DB, et al. Ventilator-induced diaphragm dysfunction (review). Journal of General Reanimatology. 2018;14(3):22.

4. Maryanova OV. Clinical and functional characteristics and non-drug correction of the syndrome of fatigue of the respiratory muscles in patients with bronchial asthma Clinical and functional characteristics and non-drug correction of the syndrome of fatigue of the respiratory muscles in patients with bronchial asthma. Scientist step Cand honey sciences. 2002:21.
5. Novik GA. Spirometry and peak flowmetry in children with bronchial asthma (practice of evaluation and monitoring): textbook. Allowance. Under the editorship. In: IM Vorontsova, editor. 2005:67.

6. Aleksandrova NP. The relative contribution of the muscles of the chest and diaphragm to the work of breathing with a resistive load. Phisiol Journal. 1993;(11):64-69.

7. Aleksandrova NP, Isaev GG. Central and peripherical components of respiratory muscle fatigue inder respiratory resistive load. Phisiol Journal. 1990;76(5):658-657.

8. Alexandrova NP, Isaev GG. The functional ability of the diaphragm with a resistive inspiratory load .Functional organization of the respiratory center and its connection with other systems: Kuibyshev State honey. Institute. Kuibyshev. 1990;105-109.

9. Barer AS, Breslav IS, Isaev GG. Phisical performance and functions of the cardiorespiratory system with additional resistance to breathing. Cosmic biology and aerospace. The medicine. 1990;24(5):20-22.

10. Aleksandrov AL, Perley VE, Gichkin A, et al. The relationship of the functional state of the diafragn with indicators of the function of external respiration on patients with COPD with severe and moderate severity. Journal Scientific notes. St. Petersburg State Medical University named after IP Pavlova. 2012;XIX(3):66-69. 